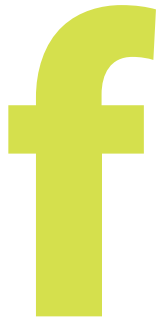

\title{
La cogestión adaptativa nos puede ayudar a enfrentar el cambio climático
}

\section{Puntos Clave}

- La cogestión adaptativa es un enfoque participativo que vincula actores forestales, empodera a las comunidades locales y sus subgrupos y fortalece las capacidades de adaptación.

- Para enfrentar al cambio climático, se necesita comunidades forestales que se comprometan a llevar a cabo esfuerzos en mitigación y adaptación a nivel global.

- La serie ACM de libros y manuales brinda orientación, así como estudios que investigan esfuerzos llevados a cabo en el pasado que podrían ser de utilidad a la hora de diseñar enfoques viables que puedan ser utilizados en la adaptación y mitigación del cambio climático a nivel local.

Por lo general, los habitantes de los bosques tropicales cuentan con pocos recursos económicos, poder o educación. Con frecuencia padecen serios problemas de salud. Sus sistemas culturales están siendo atacados. Sin embargo, estas personas tienen la misma inteligencia, compromiso y capacidades que todos tenemos. Ellos conocen, mejor que nadie, su cultura, sus intereses y metas. Teniendo esto en cuenta, la cogestión adaptativa tiene como objetivo reconocer, desarrollar $\mathrm{y}$ fortalecer las capacidades de la gente local para que

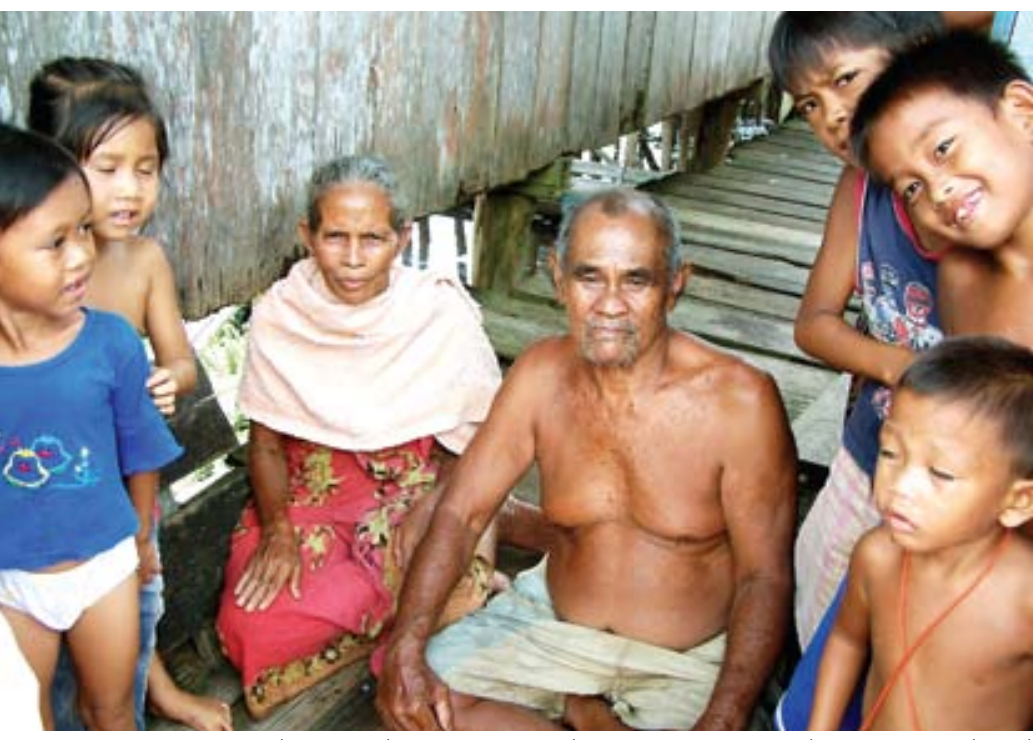

Pescadores en el Parque Nacional Danau Sentarum, Kalimantan Occidenta (Foto: Carol J. P. Colfer) puedan enfrentar los desafíos que resultan de sus ambientes cambiantes.

Inicialmente, los investigadores de CIFOR diseñaron el Programa de Cogestión Adaptativa (ACM por sus siglas en inglés) en reconocimiento de la ubicuidad del cambio, la sorpresa y la incertidumbre. Nuestras observaciones indican que los enfoques tradicionales de manejo gubernamental "de comando y control" no están funcionando y que, en realidad, no podrían funcionar.

A medida que el programa ACM fue evolucionando, se hizo evidente la necesidad de contar tanto con instituciones locales más fuertes como con mejores vínculos entre comunidades y actores que operan en otras escalas.

\section{El programa de cogestión adaptativa (ACM) y el cambio climático}

La concienciación, a nivel global, sobre los posibles impactos del cambio climático ha hecho más imperativas las necesidades antes identificadas y creado posibles oportunidades de financiamiento para satisfacerlas. Esto puede representar una oportunidad única en el tiempo, ya que existe un creciente reconocimiento de las responsabilidades que los más privilegiados tienen para enfrentar las desigualdades estructurales que contribuyen al problema (pobreza, patrones de consumo, mercados injustos). Muchos reconocen la particular vulnerabilidad al cambio climático de las poblaciones que se encuentran en desventaja. El Grupo Intergubernamental de Expertos sobre el Cambio Climático (IPCC por sus siglas en inglés) reconoce, entre otros, la importancia de fortalecer la capacidad de adaptación que tienen las personas para 'moderar riesgos potenciales, obtener los máximos beneficios de las oportunidades o para hacer frente a las consecuencias'. Si bien, inicialmente, las actividades de investigación desarrolladas por el programa ACM no abordaron el tema del cambio climático en forma específica, estos descubrimientos son particularmente pertinentes, dada la necesidad de catalizar la acción humana a todo nivel.

El reciente interés en la adaptación al cambio climático y su mitigación ha generado nuevas posibilidades, pero también presenta peligros para las comunidades que viven en los bosques y alrededor de ellos. Los debates referentes al "manejo forestal para la fijación de carbono" y REDD (Reducción de Emisiones de la Deforestación y Degradación) han puesto sobre la mesa una serie de desafíos que han sido ampliamente reconocidos, particularmente en casos donde los posibles compradores de carbono esperan negociar directamente 
con comunidades y gobiernos a nivel de paisaje. Estos desafíos, identificados por los expertos en el tema del cambio climático, incluyen:

- Instituciones y gobernanza débiles, que requieren, sobre todo,, mayor responsabilidad y transparencia, lo que a su vez exige nuevas capacidades, formas y prácticas de gobernanza.

- Problemas resultantes de las ventajas y desventajas que suponen la eficiencia, efectividad y justicia, lo que exige mayor comunicación entre los actores involucrados, el desarrollo de capacidades de negociación entre los grupos menos poderosos y una definición más clara de los derechos y responsabilidades entre los usuarios y transgresores forestales.

- Falta de visiones compartidas o planes armónicos entre las partes interesadas y la necesidad consiguiente de contar con estrategias que permitan llegar a un consenso y diseñar mecanismos conjuntos de medida y monitoreo para evaluar los avances.

- Presiones sobre la población, las mismas que pueden ser resueltas mediante la implementación de procesos que fomenten la participación directa de miembros comunitarios (especialmente mujeres) en la búsqueda de soluciones.

El programa ACM aborda estos desafíos ya que ha tenido éxito fortaleciendo la acción colectiva, el aprendizaje (y el replanteamiento de ideas), al tiempo de enfatizar la iniciativa y el empuje local. Este enfoque orientado en los procesos muestra cómo involucrar a las comunidades en las actividades que tienen como objetivo la adaptación a los cambios climáticos y disminuirlos.

\section{¿Qué es la cogestión adaptativa?}

Durante más de una década, el Programa ACM ha desarrollado actividades en una docena de países. En el siguiente cuadro se presenta la definición original elaborada por CIFOR y se incluye una modificación efectuada a la misma en el año 2008. Otros organismos, redes y personas han desarrollado ideas similares durante el mismo periodo. Estos y otros actores también han elaborado materiales útiles, basados en premisas similares sobre las capacidades que las comunidades tienen de evaluar proactivamente los problemas que ellas consideran importantes.

Desde un punto de vista filosófico, el programa ACM se basa en inquietudes e ideales democráticos en torno a aspectos como justicia y equidad, reconociendo la importancia del poder y tratando de democratizar los campos de juego mediante procesos de empoderamiento.

Contempla tres temas:

- Un tema horizontal, donde los actores de un bosque particular trabajan juntos para alcanzar metas comunes, abordando y resolviendo temas de interés relativos al bosque y las personas que viven en él y en sus alrededores,

- Un tema vertical en el que las comunidades y actores locales de otras escalas diseñan mecanismos eficaces que permiten la comunicación, cooperación y resolución de conflictos bi-direccional y,

- Un tema 'repetitivo' o progresivo donde los actores aprenden, con el tiempo, a manejar sus recursos y sus comunidades en el curso de acciones que resultan de esa creciente comprensión.

\section{Cogestión Adaptativa - \\ Definición original de CIFOR, Plus}

Primera versi囚n (2002): La cogestión adaptativa es un enfoque de valor agregado, donde la gente que tiene interés en un bosque específico decide actuar en forma conjunta para planificar, observar y aprender de la implementación de sus planes, a la vez que reconocen que, con frecuencia, no logran alcanzar los objetivos planteados. En el programa ACM estos grupos llevan a cabo serios esfuerzos por comunicar, colaborar, negociar y buscar oportunidades para aprender en forma colectiva sobre los impactos de sus acciones.

De囚nici冈n suplementaria (2008): El trabajo con un grupo específico de personas requiere que se fomente la participación de otras personas que trabajan en escalas diferentes, usualmente en un nivel más bajo o mas alto (por ejemplo, grupos de usuarios dentro de una comunidad y funcionarios distritales en niveles más altos, como es el caso en Zimbabue, Nepal, Indonesia y Filipinas). Una facilitación eficaz puede actuar como un catalizador que empodere a las comunidades para que estas mejoren sus condiciones, tanto a nivel humano como ambiental.

\section{¿Qué resultados ha tenido el programa?}

Los resultados más generales que pueden interesar a los diseñadores de políticas son las capacidades fortalecidas de las comunidades y los gobiernos locales, las mismas que permitirán que las poblaciones enfrenten tanto las nuevas oportunidades como los peligros que implican los esfuerzos de mitigación y adaptación a otras sorpresas que resultarán del cambio climático.

Debido a que estas actividades y metas están diseñadas para contextos y participantes específicos, cada lugar de trabajo ha tenido diferentes resultados. Sin embargo, por lo general, se pueden apreciar mejoras en las siguientes capacidades a nivel local: análisis de la situación, planificación, coordinación, implementación, monitoreo, negociación, gestión de conflictos, facilitación, elaboración de propuestas y otro tipo de documentos así como desarrollo de contactos.

Se aprecian mejoras en el entendimiento que la gente tiene acerca de las opiniones de otros actores, en las capacidades de actuar colectivamente y aprender de sus errores, y negociar eficazmente con actores poderosos. También se puede apreciar la existencia de definiciones, más amplias, de liderazgo a medida que las personas van reconociendo que un liderazgo eficaz incluye asimismo escuchar y recoger opiniones diversas en lugar de ser directivas y determinantes.

Pero los éxitos del programa van más allá de lo psicológico. La siguiente lista ofrece una variedad de ejemplos concretos:

- Baru Pelepat (en Jambi, Sumatra) designó un área de 900 hectáreas en su territorio tradicional para un 'bosque consuetudinario'. Los funcionarios comunitarios, distritales y provinciales la han reconocido ahora (en un contexto donde el gobierno central generalmente controla los bosques) y los lugareños han acordado reglas de gestión y las han legalizado y puesto en práctica. En una comunidad 
cercana, los miembros de un grupo de usuarios lograron impedir actos de corrupción por parte de su dirigente en las negociaciones con niveles más altos del gobierno y con una empresa de aceite de palma y negociar un mejor contrato para su comunidad, reduciendo el control de grandes empresas e individuos poderosos.

- En Nepal, actores comunitarios, distritales y nacionales empezaron a interactuar y planificar conjuntamente y de manera constructiva la gestión del bosque así como otros asuntos. Este esfuerzo tuvo como resultado la creación del Grupo de Aprendizaje de Política Nacional, el mismo que ha logrado influenciar la política forestal nacional al incorporar opiniones y experiencia por medio de consultas multipartitas en varios niveles (por ejemplo, permitiendo la formación de grupos de usuarios en el terai rico en bosques).

- En Gokwe, Zimbabue, se creó un grupo local de mujeres con el objetivo de encontrar una solución a sus problemas de manejo relacionado con la paja para escobas. Mediante simulación participativa y otros métodos derivados del programa $\mathrm{ACM}$, las mujeres analizaron la situación y, entre otras cosas, desarrollaron un método sostenible para el manejo de cosechas de paja para escobas (una fuente de ingreso adicional para familias pobres), y resolvieron problemas relacionados con la comercialización al crear diseños mas atractivos y fortalecer sus vínculos con los funcionarios del distrito.

- En Bolivia, la comunidad indígena de Cururú diseñó un plan de manejo forestal con la ayuda de ONG locales. Se creó un sistema transparente de monitoreo para controlar salarios y otros costos, a la vez que se formaba una empresa forestal comercial de propiedad de la comunidad. Esto les permitió evitar conflictos relacionados con el súbito ingreso de dinero en efectivo y mantener una empresa que proporciona empleos a los residentes y financia proyectos de desarrollo en la comunidad. El monitoreo del impacto del aprovechamiento también ha aumentado la preocupación en torno a la regeneración forestal (protegiéndose, sobre todo, la caoba para la regeneración de esta especie que, prácticamente, se ha extinguido a nivel local).

Además de estos resultados prácticos en el campo - especialmente útiles en el contexto del cambio climáticola investigación comparativa realizada en varios países ha permitido que los investigadores lleven a cabo investigación con resultados prometedores. Estos han incluido un mayor énfasis en los vínculos entre lo comunitario, distrital y nacional y el establecimiento de redes como base para el empoderamiento; comparaciones entre lugares de estudio para aprendizaje social, monitoreo y enfoques tendientes a mejorar el patrimonio; una variedad de usos para la simulación (participativa y de otro tipo); y un amplio repertorio de casos de descentralización en el mundo.

Se pueden encontrar ejemplos adicionales en los materiales listados en la página 4.

\section{ACM - ¿Cómo funciona?}

Los investigadores del programa ACM empiezan sus labores realizando una serie de estudios de contexto para examinar las tendencias históricas y políticas y el estado inicial en términos de bienestar humano y salud ambiental. En estos pasos y los siguientes, el conocimiento etnográfico les permite entender cómo funcionan los diferentes sistemas socioculturales.

Por lo general, los investigadores inician sus actividades a nivel comunitario. Un método central en el enfoque ACM es la investigación-acción participativa orientada hacia el proceso (PAR por sus siglas en inglés). PAR es un proceso de colaboración,, de largo plazo, en el que grupos de personas actúan conjuntamente en ciclos iterativos de identificación de metas, análisis, planificación, implementación, monitoreo y evaluación del progreso (Véase "la lombriz"). Este enfoque requiere de una persona que tenga la capacidad de facilitar estos procesos. En ACM, este facilitador/investigador también sirve como un eje, vinculando grupos de personas, y con el tiempo, capacitándolos en las áreas que sean necesarias para fortalecer la sostenibilidad de dicho esfuerzo.

Estos facilitadores/investigadores también traen consigo un repertorio de otros métodos que van utilizando, a medida que los requerimientos de información y análisis de los participantes se van haciendo claros.

Usuarios recientes del enfoque ACM han involucrado más explícitamente actores a nivel comunitario, distrital y en ocasiones nacional (por ejemplo Bolivia, Indonesia, Nepal, Zimbabue y 6 nuevos lugares de estudio en el marco del proyecto CIFOR-ICRAF de mosaicos de paisaje). El cambio de actitudes y enfoques en los organismos de desarrollo e investigación ha demostrado ser un proceso importante aunque lento.

\section{¿Por qué necesitamos ACM ahora?}

Existe un reconocimiento creciente que muchos de los esfuerzos desplegados en el pasado para abordar problemas a nivel local han sido innecesariamente pasivos, reactivos y/o puramente técnicos. Para abordar el cambio climático de forma eficiente, será necesario trabajar con enfoques orientados en procesos que miren al futuro, reconozcan las capacidades y oportunidades locales, y desarrollen capacidades tanto analíticas como de adaptación en varios niveles.

Para motivar a las comunidades y los gobiernos locales de la forma en que realmente se necesita para llevar a cabo estos

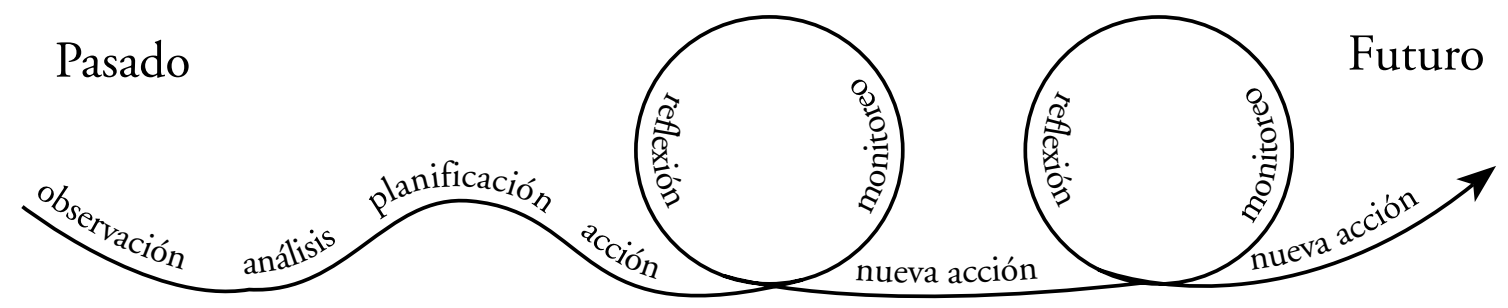

La lombriz, adaptado de Colfer 2005b 
cambios, los actores globales deben reconocer la necesidad de una respuesta clara y con sentido a las necesidades locales. Esto significa que los actores globales (organismos donantes y gobiernos en particular) deberían realizar lo siguiente:

- reconocer que estos procesos pueden tomar un lapso considerable y también indefinido de tiempo,

- tolerar mayor inseguridad y cambios en la dirección del programa debido a decisiones locales y a un mundo cambiante,

- incentivar mayor innovación, incluso riesgosa y permitir mayor flexibilidad en los planes de trabajo y actividades en todos los niveles del gobierno y,

- fortalecer las capacidades que permitan aprender de forma sistemática tanto de los éxitos y fracasos, como de la facilitación de procesos sociales en el campo.

La disponibilidad de muchos de los productos mencionados más adelante es realmente oportuna. Los análisis, manuales y consejos ofrecidos en los mismos serán invalorables para mejorar el manejo forestal que se viene llevando en la actualidad, mejorar el bienestar humano y fortalecer la mitigación y adaptación local al cambio climático. El programa ACM puede contribuir a fortalecer los esfuerzos destinados a diseñar, implementar y, cuando sea necesario, expandir estas estrategias.

Libros en inglés recientes y de próxima aparición de la Serie ACM (también disponibles en otros idiomas, consulte www.cifor.org)

Colfer, C.J.P. (ed.) 2005a. The Equitable Forest: Diversity, Community and Natural Resources. Resources for the Future/CIFOR, Washington, DC.

Colfer, C.J.P. 2005b. The Complex Forest: Communities, Uncertainty, and Adaptive Collaborative Management. Resources for the Future/CIFOR, Washington, DC.

Diaw, M.C., Aseh, T. and Prabhu, R. (eds.). Forthcoming. In Search of Common Ground: Adaptive Collaborative Management of Forests in Cameroon. CIFOR, Bogor, Indonesia.

Fisher, R., Prabhu, R. and McDougall, C. (eds.) 2007. Adaptive Collaborative Management of Community Forests in Asia: Experiences from Nepal, Indonesia and the Philippines. CIFOR, Bogor, Indonesia.

Guijt, l. (ed.) 2007. Negotiated Learning: Collaborative Monitoring in Forest Resource Management. Resource for the Future/CIFOR, Washington, DC.

Kusumanto, T., Yuliani, L., Macoun, P., Indriatmoko, Y. and Adnan, H. 2005. Learning to Adapt: Managing Forests
Together in Indonesia. CIFOR, Bogor, Indonesia.

Mandondo, A., Prabhu, R. and Matose, R. (eds.). Forthcoming. Coping Amidst Chaos: Studies on Adaptive Co-Management in Zimbabue. CIFOR, Bogor, Indonesia.

McDougall, C., Ojha, H., Banjade, M., Pandit, B.H., Bhattarai, T., Maharjan, M. and Rana, S. Forthcoming. Forests of Learning: Experiences from Research on an Adaptive Collaborative Approach to Community Forestry in Nepal. CIFOR, Bogor, Indonesia.

McDougall, C., Pandit, B.H., Banjade, M., Paudel, K.P., Ojha, H., Maharjan, M., Rana, S., Bhattarai, T. and Dangol. S. Forthcoming. Facilitating Forests of Learning: A Guidebook to Enable an Adaptive Collaborative Approach in Community Forestry User Groups. CIFOR, Bogor, Indonesia.

Moeliono, M.M., Wollenberg, E., and Limberg, G. (eds.). Forthcoming. The Decentralization of Forest Governance: Politics, Economics and the Fight for Control of Forests in Indonesian Borneo. Earthscan/ CIFOR, London.

Vanclay, J., Prabhu, R. and Sinclair, F. 2006. Realizing Community Futures. Earthscan, London.

Yasmi, Y. 2007. Institutionalization of Conflict Capability in the Management of Natural Resources: Theoretical Perspectives and Empirical Experience in Indonesia. Wageningen University, the Netherlands.

Yuliani, L., Tadjudin, D., Indriatmoko, Y., Munggoro, D.W., Gaban, F., Maulana, F. and Adnan, H. (eds.) 2007. Multistakeholder Forestry: Steps to Change. CIFOR, Bogor, Indonesia.

\section{Manuales}

Cahyat, A., Gönner, C. and Haug, M. 2007. Assessing Household Poverty and Wellbeing: A Manual with Examples from Kutai Barat. CIFOR, Bogor, Indonesia.

CIFOR. 2007. Towards Wellbeing in Forest Communities: A Sourcebook for Local Government. CIFOR, Bogor, Indonesia.

Colfer, C.J.P. 2007. Simple Rules for Catalyzing Collective Action in Natural Resource Management Contexts. CIFOR, Bogor, Indonesia.

Evans, K. and Guariguata, M.R. 2008. Participatory Monitoring in Tropical Forest Management: A Review of Tools, Concepts and Lessons Learned. CIFOR, Bogor, Indonesia.

Wollenberg, E., Anderson, J. and Lopez, C. 2005. Though All Things Differ: Pluralism as a Basis for Cooperation in Forests. CIFOR, Bogor, Indonesia.

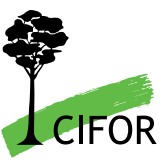

\section{ADB IDRC * CRDI DFID} Department for
International Development
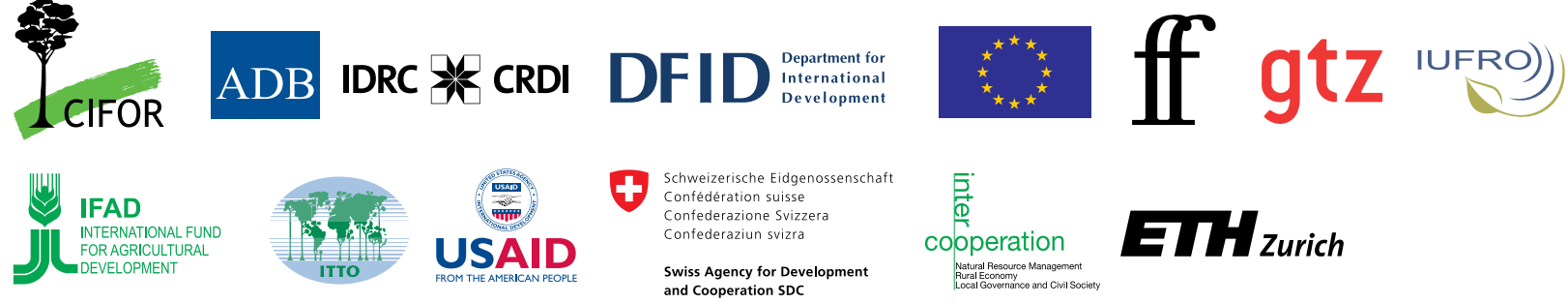

$\boldsymbol{E I H}$ Zurich

Center for International Forestry Research

Office: Jalan CIFOR, Situ Gede, Bogor Barat

16115, Indonesia

Mailing: P.O. Box 0113 BOCBD

Bogor 16000, Indonesia

\section{Tel: +62(251) 8622622}

Fax: +62(251) 8622100

E-mail: cifor@cgiar.org

Website: www.cifor.cgiar.org 